\title{
Collaboration, culture and communication: Preparing the next generation to provide rural primary health care
}

\author{
Tami L. Thomas*, Michelle Caldera, Derrick Glymph \\ Nicole Wertheim College of Nursing and Health Sciences, Florida International University, United States
}

Received: December 21, 2020

Accepted: February 23, 2021

Online Published: February 28, 2021

DOI: $10.5430 /$ jnep.v11n6p50

URL: https://doi.org/10.5430/jnep.v11n6p50

\begin{abstract}
Background and objective: In an ever-changing landscape of health care needs and demands, the ability to provide care for rural communities is often overwhelming. Rural health care in a new decade demands targeted programs to improve recruitment, training, and sustained employment of primary care providers. This project served to address rural primary healthcare needs by the development of a project model to recruit, train, educate and evaluate Advanced Practice Registered Nurse students (APRN) students who were rigorously selected for a rural traineeship and practiced in rural counties. The evaluation of preceptors was also done to assist in retention and increased numbers of rural preceptors and clinical sites. This program was designed collaboratively and implemented with rural community partners and rural healthcare leaders.

Methods: Graduate nursing students completed both a paper application and in person interviews to be selected for a rigorous 16-week clinical traineeship in the rural communities. Qualitative data were collected during interview and feedback sessions during their traineeship. Quantitative Data were collected on Process and Outcome Measures focused on learning objectives during their rural traineeship. These data were analyzed and evaluated to measure the effectiveness of program goals, outcomes, and sustainability of the program.

Results and conclusions: The results support the structured process for selecting graduate students and with an innovative program design focused on rural culture and accessing resources for patients in these same rural areas. Both students and preceptors had improved performance and satisfaction over time. The results provide a road map to recreate programs with high clinical impact and increasing numbers of rural healthcare providers. Current follow-up data with APRN post program completion demonstrated increase in employment within rural areas post-graduation.
\end{abstract}

Key Words: Collaboration, Culture, Communication, Rural primary health care

\section{INTRODUCTION}

Over the past fifteen years, work force issues have been raised regarding the availability and specialization of healthcare providers and their training for vulnerable families attempting to access primary care in rural areas. ${ }^{[1]}$ The gen- eral shortage of primary care providers is well documented within the United States and training of Advanced Practice Registered Nurses (APRNs) has attempted to reduce this shortage. ${ }^{[2,3]}$ One challenge is that the rural communities often do not have access to providers who reflect their race,

\footnotetext{
${ }^{*}$ Correspondence: Tami L. Thomas; Email: tthomas@fiu.edu; Address: Nicole Wertheim College of Nursing and Health Sciences, Florida International University, United States.
} 
ethnicity or culture. Many patients in these rural communities do not always speak English and are culturally different from many of their healthcare providers. In an effort to close this gap, APRN practice leadership in a college of nursing sought to link with rural communities, including rural healthcare clinics and county health departments, to address the shortage of healthcare providers in these underserved rural counties while also providing APRN clinical rotations and education.

\subsection{Background and significance}

Barriers to recruit rural primary care health care providers, especially APRNs, include salary, inadequate office space, limited peer network, restricted practice climate where APRNS often don't practice to their full scope as a result of a lack of the APRN role by other professionals. ${ }^{[4]}$ Retention strategies for rural APRNs and other healthcare providers includes improvement in work environment, professional autonomy, job variety, counseling to deal with stress and peer-feedback. ${ }^{[5]}$ In rural settings, preceptorship and a positive influence from APRN faculty inspire APRN nursing students to work in these often forgotten and underserved areas. ${ }^{[6]}$

Moreover, gaps in rural primary care remain unchanged and the success has been limited. After working in rural counties on vaccination education, a further collaboration was discussed with rural healthcare leaders, community members and medical staff. As a result, in 2017, a program model was developed in collaboration with rural partners from two rural counties. These collaborations included strategies for recruiting APRN students, their selection as trainees, a match with a preceptor and a specific clinical rotation in a rural clinical primary care sites. These APRN students were required to live in the community and take part in community events such as health fairs and health screenings for farm workers. This academic and clinical training would take place over 16-20 weeks and the selected trainees were paid a stipend and their tuitions were covered by funding from a Health Resources \& Services Administration (HRSA) grant.

The fact that the health disparities in these rural, underserved areas affect a wide array of age groups speaks to the need for primary healthcare providers with a broad knowledge base of age-related diseases, treatments and management protocols. After community engagement activities that spanned almost two years, an innovative project model was developed in collaboration with faculty, physicians, healthcare leaders and clinic staff to frame an innovative rural APRN academic educational model to support APRN nursing traineeships in both Hendry and Glades counties. We describe the disparities of these rural counties below.

Published by Sciedu Press

\subsubsection{Hendry county}

Hendry County was ranked last out of the entire 65 counties in Florida for social \& economic factors. ${ }^{[8]}$ The percent of people living below the poverty level in Hendry County is nearly double the average of Florida as a whole. ${ }^{[9]}$ This holds true for the percentage of children 0-17 years of age who are under the poverty level; that rate is $25.5 \%$ for Hendry County compared to $15.5 \%$ for the state. ${ }^{[9,10]}$ The average annual income for a family of four residing in Hendry County is estimated at \$37,000 annually compared to Florida's average annual household income of $\$ 51,000 .{ }^{[8]}$ A significant number of deaths in Hendry county are premature and preventable. ${ }^{[8]}$ Women over 18 years of age were less likely than other women across the state to report they had received a Pap test in the last year, at a rate of $47.9 \%$ versus $57.1 \%$ statewide. ${ }^{[8]}$ This rate is far lower than the rate in 2007 when it was $64.0 \%$, and rates of annual Pap testing are lowest for women who make under $\$ 25,000$ annually. The 3-year rate of sexually transmitted infection (STI) cases (which include chlamydia, gonorrhea and infectious syphilis) for Hendry County from 2015 to 2018 is 522 per 100,000 individuals, higher than the state average of 467 per 100,000 individuals for this time frame. ${ }^{[11]}$ Hendry County was hit hard by the economic downturn, with the unemployment rate jumping from 7.2 percent in 2000 to 14.7 percent in 2010, higher than the state rate of 11.3 percent. $^{[8]}$

In a recent Hendry County health assessment, 86. 4 percent of adults reported that they were unable to see a doctor at least once in the previous year due to cost. Also, the county had nearly double the state rate of uninsured individuals with $27.2 \%$ compared to $16.3 \%$ overall in the state of Florida. ${ }^{[9]}$ The most frequently cited reason that keeps Hendry County residents from seeking medical treatment is lack of health insurance and/or lack of ability to pay, which was more than the responses of all of the other options combined. ${ }^{[9]}$

\subsubsection{Glades county}

The percentage of all people living under the poverty level in Glades County is $20.1 \%$ higher than the state average, $15.5 \% .^{[12]}$ Lack of health insurance coverage is a significant barrier to accessing needed healthcare. ${ }^{[13]}$ In 2019, it was estimated that 25.9 percent of adults were without health insurance in Glades county as compared to a rate of 16.3 percent of Florida as a whole. ${ }^{[11]}$ The most frequent causes of death for people are cancer and heart disease, together accounting for more than 45 percent of the deaths in 2014 in Glades County. ${ }^{[14]}$ Lung cancer and congestive heart failure are the most common preventable causes of hospitalizations for residents. ${ }^{[8]}$

Socioeconomic disparities and health conditions persist in 
many rural communities; our rural partners face them every day. Communities like those served by the rural clinics in Hendry and Glades counties experience substandard access to health care and poor health outcomes. ${ }^{[7]}$ Such poor social, economic and environmental conditions have had detrimental effects on the health and well-being of these communities. Nonetheless, dedicated physicians, APRNS, Physician Assistants and nurses work every day with dedication and determination to serve these families with compassion, caring and evidenced based clinical skills.

\subsubsection{Project model}

The healthcare leadership from Hendry and Glades counties played key roles in assisting with the development of the project model. Their experience with healthcare disparities in their own rural communities served as the foundation to build an innovative project model to educate APRN students in the context, culture and practice in their rural clinics. The project team utilized extensive experience in rural community engagement, APRN program development, evaluation of process and outcome measures, and use of the Plan Do Study Act (PDSA) cycle. ${ }^{[15,16]}$ The collaborative discussions led to an innovative project model that consists of five dimensions. These dimensions are: 1) a strategy for selection and placement of APRN students in clinical sites that serve rural underserved populations; 2) expansion of clinical placements for APRN students; 3) strategies to ensure rural clinical preceptors will be recruited, oriented, evaluated and retained; 4) rapid quality improvement strategies such as the PDSA cycle are implemented to test the effectiveness of clinical training and 5) implementation of evidence-based, competency-based assessments and clinical evaluations tools to assess student performance and readiness to practice for APRNS who will serve rural communities.

This project model is framed on evidenced based strategies to retain primary care providers in underserved areas. ${ }^{[17,18]}$ The program model utilized both process and outcome measures specifically for these clinical traineeships, which tracked APRN student progress, preceptor satisfaction, and all academic programmatic requirements. This quantitative data was analyzed and monitored to ensure the goals of the rural traineeship program were met. Our collaborative efforts including our rural partners, preceptors, APRN nursing faculty led to the final project model that was tested throughout the four years the program was implemented. APRN students were well prepared to provide safe, quality care within the complex practice-based environment of the nation's evolving healthcare system. ${ }^{[19]}$ The five dimensions are depicted in Figure 1.

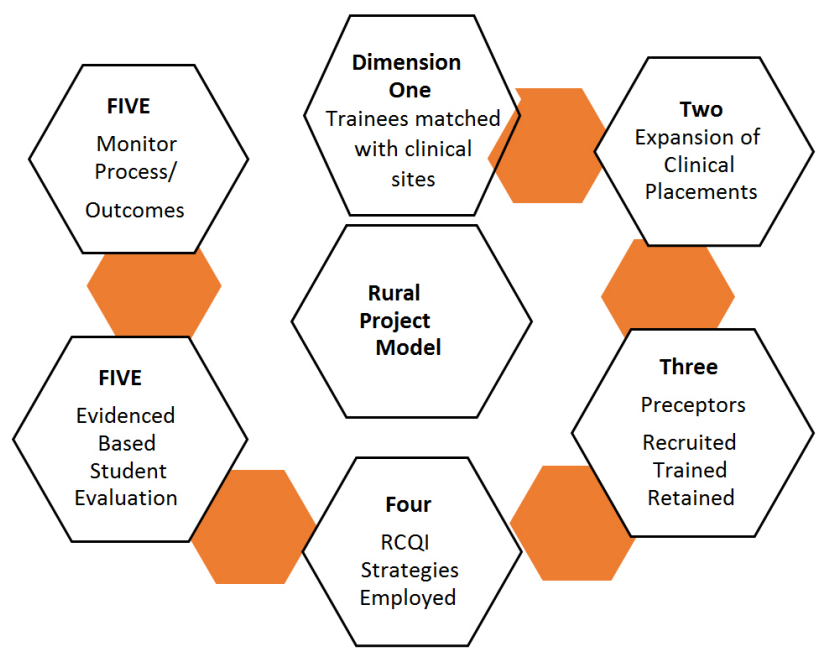

Figure 1. Developed collaboratively with rural partners and adapted from Murray and James ${ }^{[19]}$

Dimension One - Student Recruitment Process: A strategy for placement of 16 APRN trainees over 4 semesters in clinical sites was completed with our rural partners. All ARPN graduate students were eligible to apply and were recruited via pamphlets, in person recruitment during the first semester of graduate study and through a program site launched on the college website. Interested APRN students applied initially by completing a written application followed by a face-toface interview with the project director, graduate nursing faculty and rural community partners. Rural partners reviewed both the written application and participated in every student interview along with program director, program coordinator and graduate nursing faculty. This allowed for true collaboration and communication with partners months in advance - a practice that has predicted the most success in our experience. Factors considered were prior experience in serving rural communities, willingness to travel, willingness to stay overnight in a hotel as needed and previous experience volunteering in rural clinics. If the student was bilingual (English-Spanish) this was also considered but never a qualification for placement.

Selected APRN students received a traineeship award that covered tuition costs, travel expenses, and a stipend. Once selected, the APRN rural trainees were announced via email and through newsletters and at College wide meetings. The intent was to remind everyone involved that selection, as well as serving rural communities was an honor and a privilege. APRN students were supported by faculty and program staff with one-on-one mentoring that included weekly face-to-face meetings as needed. This one-on-one support of a nursing mentor has been reported as key predictor of academic progression and future success in nursing. ${ }^{[20]}$ In addition, the 
project coordinator traveled weekly to the rural clinics to evaluate and address any student, preceptor, partner or practice concern.

Dimension Two - Expansion of clinical placements for APRN students were planned and achieved. This expansion led to an increase in clinical placements as our rural partners interviewed and sought to hire our rural APRN trainees who had been placed in these rural underserved areas. Also, additional rural clinical preceptors volunteered as the program progressed and the caliber of APRN students, the weekly support and evaluation demonstrated our commitment to these rural clinics.

Dimension Three - Implementation of strategies to ensure clinical preceptors were recruited, oriented, evaluated, and retained was achieved during routine visits prior to implementing the model to recruit preceptors during the first 6 months program implementation. They also established a personal one-on-one relationship with providers who volunteered to be clinical preceptors. This was essential to establish trust and ensure the quality of APRN students assigned to their rural primary care clinics.

Dimension Four - In dimension four, rapid quality improvement strategies were implemented to test the effectiveness of clinical training with the systematic tracking of clinical experiences, management of student debriefing and weekly evaluation that included the collection of both process and outcome measures and the programmatic requirements of mid and final semester evaluation of each APRN student. PDSA were utilized in each dimension of the project model; but in the evaluation of process and outcome measures, quantitative data were routinely used to implement changes and enhancements, resulting in success for both the APRN student and the rural preceptor.

Dimension Five - This dimension contained evaluation components of process and outcomes for both nurse practitioner students and preceptors. The implementation of evidencebased, competency-based assessments and clinical evaluations tools gauged student performance and readiness to practice. This contributed to optimal student outcomes and successful employment of primary care nurse practitioners in rural and underserved areas. In addition, both students and preceptors were made aware of an additional adapted model from Diekelmann and Barragan with constructivism provided as a visual guide for the enhancement of APRN education and development of future APRN Faculty Preceptors, see Figure 2. ${ }^{[18,21,22]}$

\section{Pedagogy Model}

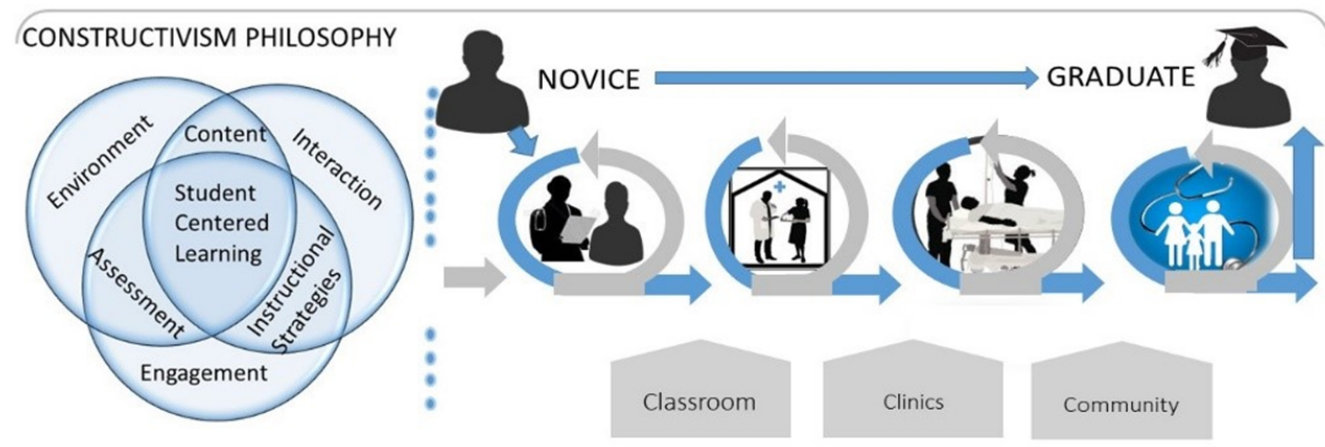

Figure 2. Adapted pedagogy model utilized for preceptor and graduate nursing traineeship encompasses multiple factors associated with positive interaction of classroom learning and clinic application impact on community. ${ }^{[21]}$

Through the implementation of the project model, APRN students engaged with rural and vulnerable communities served by our primary practice partners in Hendry and Glades counties. The environment and the rural clinics substantially enhanced APRN education framed upon the project model. This project served to address rural primary healthcare needs by the development of a project model to recruit, train, educate and evaluate Advanced Practice Registered Nurse students (APRN) students who were rigorously selected for a rural traineeship and practiced in rural counties. The evaluation of preceptors was also done to assist in retention and increased numbers of rural preceptors and clinical sites.

\section{Methods}

This descriptive study utilized methods built upon evidencedbased pedagogy and included continuous preceptor and faculty development on innovative teaching strategies to enhance student clinical leadership skills, team building and 
collaborative problem-solving, culturally appropriate care, integration of telehealth and Information Technology. Based on clinical partner feedback, rural clinical capacity, and available preceptors, this program aimed to place a total of 16 APRN trainees. Clinical placement of selected ARNP students took place over 4 semesters with small cohorts of 3-5 students assigned to a rural clinical site throughout a 16-week period. Prior to collecting any data on APRN students or preceptors, Institutional Review Board (IRB) approval was obtained by the University. After Institutional Review Board approval, quantitative measures based on specific process and outcome measures were used to collect data at different times during the project.

Outcome and process measures for APRN students and preceptors were based on review of the literature with priority to those items that align with the Benner Theory of Novice to Expert and the Synergy Model of Nursing. ${ }^{[5,23]}$ Process measures items were derived from standardized improvement approaches. ${ }^{[24,25]}$ Outcome measures items were selected to align with domains and core competencies of advanced nursing practice as supported the by the National Organization of Nurse Practitioner Faculties (NONPF) and widely used evidenced based practice standards. ${ }^{[15,22]}$

This quantitative data was collected using responses to Process Measures and Outcome measures on a Likert scale of 1 through 5 with the following choices: N/A for No opportunity to perform or not observed; 0 -Omits required critical element; 1 Requires extensive prompting to perform behavior- obtains relevant data $30 \%$ of the time; 2 Requires much prompting to perform behavior- obtains relevant data $50 \%$ of the time; 3 Requires moderate prompting to perform behavior- obtains relevant data $70 \%$ of the time; 4 Requires minimal prompting to perform behavior- obtains relevant data $90 \%$ of the time or 5 Performs behavior independently clinical autonomy.

Student process measures included 4 daily measures per student and these focus on how the APRN student prepared for and completed their daily clinical experience. Student outcome measures include 39 measures per APRN student, per week, and focus on specific outcomes related to the core curriculum.

Process and Outcome measures for all rural preceptors were also collected weekly to evaluate and guide interactions with their respective APRN student preceptee. Rural preceptor process outcomes were focused on the preceptor's preparation interaction with the APRN graduate students such as specific patient or family care, including management of chronic illness. Preceptor outcome measures focused on preceptor provision of quality opportunities for APRN graduate students, evaluation of critical thinking, patient assessments, diagnosis, treatments and anticipatory guidance/health promotion instructions were evidenced based. The measurement and evaluation of these behaviors were key to the success of both the rural preceptor and the APRN graduate students. We structured these preceptor outcomes based on findings from a recent study that identified a knowledge gap of preceptors' self-evaluated competence and a gap in preceptor confidence in determining the match between the rural preceptor and the APRN graduate student trainees they would precept during the rural traineeship. ${ }^{[26]}$ The scoring of preceptor process and outcome measures is as follows: N/A - no opportunity, 1-Never Occurs, 2-rarely occurs $0 \%-39 \%$ of the time, 3-Occurs Sometimes 40\%-69\% of the time, 4-Usually Occurs $70 \%-89 \%$ of the time and 5-Occurs $90 \%-100 \%$ of the time.

The methods and tools used to evaluate student progression also included the student centric evaluation required by the APRN program, completed at mid-point and the end of the semester. Data was collected after each clinical rotation encompassing quantitative and qualitative approaches that monitor student care, progress, clinical competency and the effect of preceptor mentorship. ${ }^{[7,16]}$ For the observation component of this project, qualitative data was collected via anonymous surveys analyzing students' and preceptors' experience in rural clinics. Students were given one-on-one feedback by preceptors before, during and after each clinical day. While students were expected to apply the knowledge, practice skills and demonstrate professional attitudes of professional nursing practice, the rural traineeship program is for student learning and not for a "performance" experience. All APRN students written and verbal comments were included as qualitative feedback as part of the anonymous surveys given at the end of the rural traineeship program. Specific project methodology allowed for student and preceptor evaluation based on increase in student clinical performance and feedback from their preceptor. These were linked to student overall clinical experience in a rural health setting and demonstrated likelihood of pursuing a career in a rural clinic.

\section{Analysis}

All statistical analyses were performed using SPSS 17.0. Statistical significance was assessed using an alpha level of .05. Outliers were examined and correlations run on student process variables. A general linear model was run using process measures (1,728 data points) and outcome measures (7,488 points) for process and outcome measures for APRN students. This same linear model was run using within subjects' factors to verify APRN student results over time. Pairwise comparison tests and Wilks' Lambda test were completed 
with a significance of .000 . These same statistical tests were completed on preceptor process and outcome measures. The general linear model was completed, within group comparisons and pairwise comparisons at .000 significance and Wilks' Lambda significance at .003. Data was analyzed and monitored to ensure the rural traineeship was successfully implemented along with student and preceptor evaluation during rural traineeship project.

\section{RESUlts}

Results indicated completion of program with exposure to a rural health clinical setting enhanced ARNP student com- prehensive primary care knowledge, clinical autonomy, and increased their desire to work in a rural community. Descriptive statistics on both student and preceptor demographics were calculated. Student demographic results include11 $(68.8 \%)$ identified as male and $5(31.3 \%)$ Female; $12(75 \%)$ as Hispanic and $4(25 \%)$ Non-Hispanic; $4(25 \%)$ as White, $4(25 \%)$ Black, $3(18.8 \%)$ Asian, and $5(31.3 \%)$ as Other, see Table 1 . Additionally, of the 16 graduate nursing student trainees, $10(62.5 \%)$ were enrolled within the Adult nursing program, $3(18.8 \%)$ in the family program, and $3(18.8 \%)$ in the pediatric nursing program (see Table 1).

Table 1. Demographics of participants in rural project program

\begin{tabular}{|c|c|c|c|c|}
\hline \multicolumn{5}{|c|}{ Rural Program Participant Demographics } \\
\hline \multirow{2}{*}{$\begin{array}{l}\text { Individual-level } \\
\text { variables }\end{array}$} & \multicolumn{2}{|l|}{ Trainees } & \multicolumn{2}{|l|}{ Rural Preceptors } \\
\hline & Frequency $(n=16)$ & Valid Percentage (\%) & Frequency $(n=7)$ & Valid Percentage (\%) \\
\hline \multicolumn{5}{|l|}{ Gender } \\
\hline Male & 11 & 68.8 & 4 & 57.1 \\
\hline Female & 5 & 31.3 & 3 & 42.9 \\
\hline \multicolumn{5}{|l|}{ Race } \\
\hline White & 4 & 25.0 & 3 & 42.9 \\
\hline Black & 4 & 25.0 & 1 & 14.3 \\
\hline Asian & 3 & 18.8 & 1 & 14.3 \\
\hline Other & 5 & 31.3 & 2 & 28.6 \\
\hline \multicolumn{5}{|l|}{ Ethnicity } \\
\hline Hispanic & 12 & 75.0 & 2 & 75.0 \\
\hline Non-Hispanic & 4 & 25.0 & 5 & 25.0 \\
\hline \multicolumn{5}{|l|}{ Health Profession } \\
\hline Adult & 10 & 62.5 & 2 & 28.6 \\
\hline Family & 3 & 18.8 & 3 & 42.9 \\
\hline Pediatrics & 3 & 18.8 & 2 & 28.6 \\
\hline \multicolumn{5}{|c|}{ Previous Experience working in a Rural Clinical Site } \\
\hline No & 0 & 0.0 & 2 & 28.6 \\
\hline Yes & 16 & 100.0 & 5 & 71.4 \\
\hline
\end{tabular}

All APRN students have shown improvement in clinical competency and decision-making skills at the initial, mid and final evaluations, demonstrating an enormous positive impact of the program on APRN students who participated in the rural traineeship with a $.000 \mathrm{sig}$. value (see Table 2 ). These effects over time show increasing improvement. Improvement was equally distributed across gender, race, ethnicity and specialties (adult, family, and pediatrics). Results from process and outcomes indicate $100 \%$ improvement rates in students' clinical competency. Final clinical evaluation results showed $93 \%$ of students received an average overall score of 5 on the category "performs behavior independently" during their final clinical assessment. There was no significant difference in trainee improvement rates among variables making this an effective rural APRN education among all specialties and demographics.

Results from preceptor process and outcomes measures completed for students demonstrated $100 \%$ of participating students believing preceptor mentorship is instrumental in engaging with patients and providing adequate care to rural communities. Similar to student trainees, rural preceptors received initial and final process and outcome measure evaluation throughout their participation in the program. Results 
from the preceptor process and outcome measures demonstrated $100 \%$ improvement rate for rural healthcare providers' clinical mentorship skills. This included an improvement in communication skills with APRN student and APRN faculty to provide clinical feedback and educate students on evidenced based practice, patient-centered care, disease processes, appropriate primary care assessment, diagnosis, and treatment plan for each patient, and culturally competent care.

Table 2. Analysis of trainee demographic on improvement of clinical competency following clinical traineeship program

\begin{tabular}{|c|c|c|c|c|c|c|c|}
\hline \multicolumn{2}{|c|}{ rive } & \multicolumn{2}{|l|}{ Mean } & \multicolumn{2}{|c|}{ Std. Deviation } & \multicolumn{2}{|c|}{$\mathbf{N}$} \\
\hline \multicolumn{2}{|c|}{ First Clinical Evaluation } & \multicolumn{2}{|l|}{147.37} & \multicolumn{2}{|l|}{10.327} & \multicolumn{2}{|c|}{16} \\
\hline \multicolumn{2}{|c|}{ Mid Clinical Evaluation } & \multicolumn{2}{|l|}{156.81} & \multicolumn{2}{|l|}{5.431} & \multicolumn{2}{|c|}{16} \\
\hline \multicolumn{2}{|c|}{ Final Clinical Evaluation } & \multicolumn{2}{|l|}{164.69} & \multicolumn{2}{|l|}{1.250} & \multicolumn{2}{|c|}{16} \\
\hline \multicolumn{8}{|c|}{ Multivariate Tests } \\
\hline \multicolumn{2}{|l|}{ Effect } & Value & $\mathbf{F}$ & Hypothesis df & Error df & Sig. & Partial Eta Squared \\
\hline \multirow{4}{*}{ Time } & Pillai's Trace & .757 & $21.770^{\dagger}$ & 2.000 & 14.000 & .000 & .757 \\
\hline & Wilks’ Lambda & .243 & $21.770^{\dagger}$ & 2.000 & 14.000 & .000 & .757 \\
\hline & Hotelling's Trace & 3.110 & $21.770^{\dagger}$ & 2.000 & 14.000 & .000 & .757 \\
\hline & Roy's Largest Root & 3.110 & $21.770^{\dagger}$ & 2.000 & 14.000 & .000 & .757 \\
\hline
\end{tabular}

Note. Design: Intercept Within Subjects Design: Time ${ }^{\dagger}$ Exact statistic

These improvements in preceptor competency positively impacted the rural APRN student educational and clinical training experience. Multivariate analysis of variance was used to examine the relationship between gender, race, ethnicity, and nursing discipline (independent variables) and the rate improvement of clinical competency at the initial, mid, and final intervals (dependent variable) (see Table 3). Data analysis showed no significant relationship between gender, nursing discipline, race, or ethnicity as contributing factors affecting improvement rates of clinical evaluation. This serves to show that this clinical traineeship program is an effective and efficient replicable model for increasing clinical competency and decision-making skills for all nursing students. This clinical program is applicable across all nursing disciplines and serves as a culturally competent comprehensive model of clinical learning.

Table 3. Analysis of trainee demographic on improvement of clinical competency following clinical traineeship program

\begin{tabular}{lllllll}
\hline Between Subjects Test & \multicolumn{1}{l}{} & & & & \\
\hline & Sum of Squares & Df & Mean Square & F & Sig. & Partial Eta Squared \\
\hline Nursing Discipline & 258.605 & 2 & 129.302 & 1.252 & .378 & .385 \\
Gender & 21.489 & 1 & 21.489 & .208 & .672 & .049 \\
Race & 268.003 & 2 & 134.017 & 1.297 & .368 & .393 \\
Ethnicity & 215.083 & 2 & 110.780 & 1.072 & .520 & .747 \\
\hline
\end{tabular}

Note. Dependent Variable: Improvement of clinical competency following completion of clinical program

These results support the observation that, overtime, preceptors have shown improvement in preceptorship skills and that the APRN rural traineeship program has had a positive impact on their precepting of APRN students, with a .003 sig. value. in outcome measures and .004 in process measures. Again, improvement was equally distributed across medical profession, gender and previous precepting history. Gender and discipline (pediatrician, physician and nurse practitioner) were not influential in the rate of improvement.

Following completion of rural traineeship program, partic- ipating APRN students were asked to provide feedback regarding their clinical experience as well as health perceptions of rural communities. Qualitative data from feedback demonstrated positive clinical experiences for $100 \%$ of students following completion of traineeship program. The qualitative comments of the APRN grad student trainees were collated using qualitative methods and analyzed to identify themes. The themes included : 1) Completion of the rural traineeship program increased their desire and readiness to work with rural and underserved communities 2) Exposure 
to a rural healthcare setting further developed APRN nursing career skills by improving clinical competency, cultural sensitivity, and overall clinical care and treatment, and 3) despite the challenges of temporarily living in the community or commuting for over three hours, working in rural communities can further enhance clinical competency and overall satisfaction with the role of the Advanced Practice Registered Nurse.

The 2020 generation of nurse practitioner students reported challenges to increasing provider availability in rural areas as: travel distance to clinic location, remoteness, salary and incentives and culture change. Our local clinical partners reported that, even with hiring the nurse practitioners who participated in this program, there is still an overall need to increase provider availability. Our results on preceptor preparedness indicated an opportunity to improve mentors' competence, particularly through enhancing organizational mentoring practices and relevant resources in clinical environments. These positive experiences associated with participation in the program further impacted the overall health care for patients, since all students stated that throughout their rural clinical traineeship, they felt a gradual improvement in the care they provided patients.

\section{Discussion}

This four-year program allocated effective and efficient solutions for healthcare limitations in rural communities by preparing APRN students and increasing the availability of rural nurses. ${ }^{[1,22,25]}$ The project model utilized within this study relied on community collaboration and innovations in clinical training for ARNP's to increase efforts in rural areas. Utilization of the programs 5-dimension model was critical in the success of the program's implementation and ARNP student clinical performance. Clinical performance and a positive experience with preceptors increased the desire to work in rural areas. Tailored recruitment and placement of ARNP students with specific rural preceptors allowed for specialized clinical training and performance feedback that enhanced clinical decision making, clinical autonomy, and knowledge to address a variety of health concerns specific to rural patients.

Data from student clinical assessment and feedback from preceptors support the idea that this model can be replicable in other rural areas. Our data and overall outcomes support earlier findings from similar studies that suggest students with urban backgrounds who complete clinical rural practice are more likely to seek clinical employment in rural settings. ${ }^{[27]}$ Moreover, this data highlights the important association between positive clinical experience and the impact on overall provider care and patient treatment which have been keys

Published by Sciedu Press to ongoing positive relationships with clinical preceptors. ${ }^{[6]}$ Analysis from other studies indicated that nurse practitioner students' clinical practice assessments already include many good practices, but when preparing this future generation of nurse practitioners who will practice in rural areas, this innovative project model as described will lead to competence in rural primary care practice areas. ${ }^{[7,17,18]}$

Utilizing data to evaluate and shape future rural APRN clinical/educational practice in rural settings is just as important as utilizing data to continually improve standard APRN clinical/educational practice settings. Overall, the project served to evaluate the student and preceptor progress and informed the program model in addition to analyzing and improving clinical training skills of students. The overarching goal was to develop a model that could be reproduced in rural areas, evaluate APRN students and their preceptors in clinical practice, increase the number of primary care providers in rural areas and address rural health disparities with data. ${ }^{[7]}$

\subsection{Lessons Learned}

The preparation of APRNs for rural primary care and the development of rural preceptors was achieved successfully using this project model. The use of our project model has maintained success for both APRN students and preceptors over the past four years. Nonetheless there were valuable lessons we learned along the way. First, we found the inperson interviews were essential and an indicator of overall success. Students with high interview scores were later associated with higher clinical performance and healthier professional skills with clinical staff. Second, we found that a longer orientation, at least a full day in the rural community was more helpful than any online education or literature review. Experiencing the community by eating at a local restaurant, being introduced to local healthcare leaders, prospective preceptors, planning for community outreach and attending weekend community events clarified expectations for both the students and the preceptors. Third, APRN students debriefed weekly with the program manager who visited each rural clinic on a weekly basis to resolve programmatic issues or assist with housing or weekend community outreach participation. Lastly, positive relationships and collaborations with rural clinical partners and community stakeholders provide framework for efforts to reduce rural health inequity and facilitate implementation of programs aimed to increase culturally competent provider coverage across all communities. ${ }^{[22,28,29]}$

\subsection{Limitations}

Project limitation included monetary award incentive associated with program completion on nursing student clinical 
performance, however, development of project model addressed this by implementing a detailed student selection process which included program application and interviews. Secondary project limitations included small sample size and three clinical practice sites. Both limitations were influenced by the lack of provider availability and health services in rural Hendry and Glades County. With only three available clinical sites within a 100 miles radius and limited provider availability to precept graduate nursing students, recruitment and size of trainee cohorts were specific and dependent on available resources. Small group of available and trained physicians and nurses to precept nursing students meant reduced number of accepted students to the program. Additionally, lack of available healthcare equipment and services in rural communities limited expanding program to other nursing disciplines such as psychiatric mental health or infectiology. However, despite project limitations, the ultimate project goals and objectives were achieved.

\section{Conclusion}

This innovative clinical traineeship program serves as a replicable model for globally increasing ARNP training and availability for primary care services in rural underserved communities and allocating effective and efficient solutions for healthcare limitation in rural communities. Starting from the development of this project model to its implementation, evaluation of students and preceptors, and use of continuous feedback has been a wonderful four-year journey for APRN students, rural preceptors and rural partners. Rural communities who are often overlooked and invisible are now becoming part of the fabric of graduate education in an urban university serving minorities. Graduate students now ask for rural placement because of the great conversations they have shared with their peers. As the program sunsets this coming year, rural APRN placements will continue with the hope that the model will be adapted in other areas and graduate programs in nursing who serve vulnerable populations will enjoy similar success. The future looks positive and we hope our rural communities will now have APRNs who have graduated from this program serving these rural areas. Follow-up with ARNP students who completed this program demonstrated active career placement in a rural and underserved community. Several students are already working in rural communities and share that their passion was ignited, and their confidence increased because of the rural traineeship program.

\section{CONFlicts OF INTEREST Disclosure}

The authors declare that there is no conflict of interest.

\section{REFERENCES}

[1] Donelan K, DesRoches CM, Dittus RS, et al. Perspectives of physicians and nurse practitioners on primary care practice. N Engl J Med. 2013; 368(20): 1898-906. PMid:23675658 https ://doi .org/10 .1056/NEJMsa1212938

[2] Huang ES, Finegold K. Seven million Americans live in areas where demand for primary care may exceed supply by more than 10 percent. Health Aff (Millwood). 2013; 32(3): 614-21. PMid:23426931 https://doi.org/10.1377/hlthaff.2012.0913

[3] MacLean L, Hassmiller S, Shaffer F, et al. Scale, causes, and implications of the primary care nursing shortage. Annu Rev Public Health. 2014; 35: 443-57. PMid:24422561 https://doi.org/10.1146/ annurev-publhealth-032013-182508

[4] Hain D, Fleck J. Barriers to Practice and the Impact on Health Care: A Nurse Practitioner Focus. Journal of the Advanced Practitioner in Oncology. 2014; 19.

[5] Wei H, Sewell KA, Woody G, et al. The state of the science of nurse work environments in the United States: A systematic review. Int J Nurs Sci. 2018; 5(3): 287-300. PMid:31406839 https : //doi.org/10.1016/j.ijnss.2018.04.010

[6] Oosterbroek TA, Yonge O, Myrick F. Rural Nursing Preceptorship: An Integrative Review Online Journal of Rural Nursing and Health Care. 2017; 17(1). https://doi.org/10.14574/ojrnhc.v17i 1.430

[7] Thomas T, DiClemente R, Snell S. Overcoming the triad of rural health disparities: How local culture, lack of economic opportunity, and geographic location instigate health disparities. Health
Education Journal. 2014; 73(3): 285-94. PMid:25242822 https : //doi.org/10.1177/0017896912471049

[8] Hendry County Florida Health Assessment [Internet]. Florida Department of Health. 2019 [cited November 11, 2020]. Available from: http://www.floridahealth.gov/provider-and-par tner-resources/community-partnerships/floridamapp/ state-and-community-reports/hendry-county/_documen ts/Hendry_CHA2.pdf

[9] Hendry County Quick Facts [Internet]. 2019 [cited November 11, 2020]. Available from: https : //www.census.gov/quickfacts/ hendrycountyflorida

[10] Florida CHARTS [Internet]. Florida Department of Health. 2019 [cited November 11, 2020]. Available from: http://www. flhealthcharts . com/ChartsReports/rdPage. aspx?rdReport=ChartsProfiles. CountyStateProfile

[11] County Health Rankings 2020 [Internet]. 2020 [cited November 11, 2020]. Available from: https://www. countyhealthrankings .org/sites/default/files/media/document/CHR2020_FL -v2.pdf

[12] Glades County Florida Health Assessment [Internet]. Florida Department of Health. 2016 [cited November 11, 2020]. Available from: http://www.floridahealth.gov/provider-and-par tner-resources/community-partnerships/floridamapp/ state-and-community-reports/glades-county2/_docume nts/Glades_CHA2.pdf

[13] Bennett P, Jones D, Brown J, et al. Supporting rural/remote primary health care placement experiences increases undergraduate nurse con- 
fidence. Nurse Educ Today. 2013; 33(2): 166-72. PMid:22445533 https://doi.org/10.1016/j.nedt.2012.02.015

[14] Family Health Centers of Southwest Florida Community Health Assessment. Appendix C: Hendry County Data, 2014 [Internet]. Florida Department of Health. 2014 [cited June 1, 2015]. Available from: http://www.hpcswf.com/wp-content/uploads/2011/ 12/Family-Health-Centers-Health-Assessment-2014-H ENDRY-COUNTY-DATA.pdf

[15] LaVeist TA, Pierre G. Integrating the 3Ds-social determinants, health disparities, and health-care workforce diversity. Public Health Rep. 2014; 129 Suppl 2: 9-14. PMid:24385659 https ://doi .org/10 $.1177 / 00333549141291 \mathrm{~S} 204$

[16] Wilson R, Godfrey CM, Sears K, et al. Exploring conceptual and theoretical frameworks for nurse practitioner education: a scoping review protocol. JBI Database System Rev Implement Rep. 2015; 13(10): 146-55. PMid:26571290 https://doi.org/10.11124/j bisrir-2015-2150

[17] Fatima Y, Kazmi S, King S, et al. Positive placement experience and future rural practice intentions: findings from a repeated cross-sectional study. J Multidiscip Healthc. 2018; 11: 645-52. PMid:30519033 https://doi .org/10.2147/JMDH.S178138

[18] Lea J, Cruickshank M. Supporting new graduate nurses making the transition to rural nursing practice: views from experienced rural nurses. J Clin Nurs. 2015; 24(19-20): 2826-34. PMid:26177875 https://doi.org/10.1111/jocn. 12890

[19] Murray TA, James DC. Evaluation of an academic service partnership using a strategic alliance framework. Nurs Outlook. 2012; 60(4): e17-22. PMid:22177471 https://doi.org/10.1016/j.outloo k. 2011.10 .004

[20] Hickey KT, Hodges EA, Thomas TL, et al. Initial evaluation of the Robert Wood Johnson Foundation Nurse Faculty Scholars program. Nurs Outlook. 2014; 62(6): 394-401. PMid:25085329 https : //doi.org/10.1016/j.outlook.2014.06.004

[21] Diekelmann N. Narrative Pedagogy: Heideggerian hermeneutical analyses of lived experiences of students, teachers, and clini- cians. ANS Adv Nurs Sci. 2001; 23(3): 53-71. PMid:11225050 https://doi.org/10.1097/00012272-200103000-00006

[22] Melnyk BM, Gallagher-Ford L, Long LE, et al. The establishment of evidence-based practice competencies for practicing registered nurses and advanced practice nurses in real-world clinical settings: proficiencies to improve healthcare quality, reliability, patient outcomes, and costs. Worldviews Evid Based Nurs. 2014; 11(1): 5-15. PMid:24447399 https://doi.org/10.1111/wvn.12021

[23] Benner P. From Novice to Expert. The American Journal of Nursing 1982; 82(3). https://doi.org/10.2307/3462928

[24] Reed KD, Kaplow R. The AACN Synergy Model for Patient Care: A Nursing Model as a Force of Magnetism. Nursing Economics. 2008; 26(1): 17-25.

[25] Scarpa R, Connelly PE. Innovations in performance assessment: a criterion based performance assessment for advanced practice nurses using a synergistic theoretical nursing framework. Nurs Adm Q. 2011; 35(2): 164-73. PMid:21403490 https://doi.org/10.109 7/NAQ.0b013e31820fface

[26] Pramila-Savukoski S, Juntunen J, Tuomikoski AM, et al. Mentors' self-assessed competence in mentoring nursing students in clinical practice: A systematic review of quantitative studies. J Clin Nurs. 2020; 29(5-6): 684-705. PMid:31794105 https ://doi .org/10.1 $111 /$ jocn. 15127

[27] Pront L, Kelton M, Munt R, et al. Living and learning in a rural environment: a nursing student perspective. Nurse Educ Today. 2013; 33(3): 281-5. PMid:22732124 https://doi.org/10.1016/j.ne dt. 2012.05.026

[28] Chillag KL, Lee LM. Synergistic Disparities and Public Health Mitigation of COVID-19 in the Rural United States. J Bioeth Inq. 2020.

[29] Summers-Gabr NM. Rural-urban mental health disparities in the United States during COVID-19. Psychol Trauma. 2020; 12(S1): S222-S4. PMid:32478541 https://doi.org/10.1037/tra000 0871 